\title{
Correction to: Metaheuristics in Machine Learning: Theory and Applications
}

\author{
Diego Oliva, Essam H. Houssein, and Salvador Hinojosa
}

\section{Correction to:}

D. Oliva et al. (eds.),

Metaheuristics in Machine Learning: Theory

and Applications, Studies in Computational Intelligence 967, https://doi.org/10.1007/978-3-030-70542-8

The original version of the book was published with incorrect affiliation for the author M. Hassaballah. Affiliation has been updated with correct affiliation for the following chapters:

M. Hassaballah

Department of Computer Science, Faculty of Computers and Information, South Valley University, Qena 83523, Egypt

"A Review of Metaheuristic Optimization Algorithms in Wireless Sensor Networks"

"Drug Design and Discovery: Theory, Applications, Open Issues and Challenges"

"Integration of Internet of Things and Cloud Computing for Cardiac Health Recognition"

The chapters and book have been updated with the changes.

The updated versions of these chapters can be found at https://doi.org/10.1007/978-3-030-70542-8_9

https://doi.org/10.1007/978-3-030-70542-8_15

https://doi.org/10.1007/978-3-030-70542-8_26

(C) The Author(s), under exclusive license to Springer Nature Switzerland AG 2021

C1

D. Oliva et al. (eds.), Metaheuristics in Machine Learning: Theory and Applications,

Studies in Computational Intelligence 967,

https://doi.org/10.1007/978-3-030-70542-8_31 